\title{
Ccm1 Regulates Microvascular Morphogenesis during Angiogenesis
}

\author{
Huiling Liu ${ }^{a}$ Daniele Rigamonti ${ }^{b}$ Ahmed Badr ${ }^{c}$ Jun Zhang ${ }^{c}$

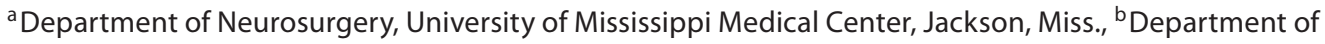 \\ Neurological Surgery, Johns Hopkins University School of Medicine, Baltimore, Md., and ' $\mathrm{COE}$ for Neurosciences, \\ Departments of Anesthesiology, Neurosurgery, and Biomedical Sciences, Texas Tech University Health Science \\ Center, El Paso, Tex., USA
}

\section{Key Words}

Angiogenesis - Cerebral cavernous malformation •

CCM1 - Intersegmental vessels $\cdot$ Vacuole/lumen formation •

Vascular endothelial cells

\begin{abstract}
Cerebral cavernous malformations (CCMs) are characterized by abnormally dilated intracranial capillaries that have a propensity to bleed. The development of some CCMs in humans has been attributed to mutations in CCM1 and CCM2 genes. In animal models, major cardiovascular defects caused by both gene mutations have been observed. However, the effects of the loss of $\mathrm{Ccm}$ function on the microvasculature in animal models are less defined. Using high-resolution imaging in vivo, we demonstrated that the loss of $\mathrm{Ccm} 1$ in zebrafish embryos leads to failed microvascular lumenization during angiogenesis due to impaired intraendothelial vacuole formation and fusion. No developmental changes during vasculogenesis and the initial stage of angiogenesis were observed, being in contrast to prior reports. In vivo zebrafish studies were further substantiated by in vitro findings in human endothelial cells that elucidated the biochemical pathways of CCM1 deficiency. We found that CCM1 regulates angiogenic microvascular lumen formation through Rac1 small GTPase. In summary, $\mathrm{Ccm} 1$ has been identified as a key angiogenic modulator in microvascular tubulogenesis. Addi-
\end{abstract}

tionally, the microvascular pathology observed in developing $\mathrm{Ccm} 1$ mutant zebrafish embryos mirrors that seen in human CCM lesions, suggesting that zebrafish might provide a superior animal model to study the pathogenesis of human CCM.

Copyright $\odot 2010$ S. Karger AG, Basel

\section{Introduction}

In humans, cerebral cavernous malformations (CCMs) are vascular lesions characterized by distended intracranial capillaries with a thin layer of vascular endothelial cells (VECs). This vasculature lacks supportive parenchyma, compromising vascular integrity and thus potentially causing hemorrhage and stroke. Hereditary CCM has been found to be caused by a loss-of-function mutation in one of three CCM genes: KRIT1 (CCM1), MGC4607 (CCM2), and PDCD10 (CCM3). On the molecular level, CCM1 interacts with integrin cytoplasmic domain-associated protein- $1 \alpha$ (ICAP1 $\alpha$ ), suggesting a role in $\beta_{1}$-integrin-mediated signaling [1-3]. Researchers have established that all three CCM gene products interact with each other and form a CCM complex [4-7] that modulates signal transduction pathways $[3,8,9]$. While the precise pathophysiology connecting the CCM protein complex to microvascular malformation has remained 
elusive, the critical importance of the complex is evidenced by the observation that at least one of its components is disrupted in most human CCMs. Therefore, much attention has recently been directed toward the roles played by the CCM complex in vasculogenesis and/ or angiogenesis.

In contrast to humans, defects in major blood vessels and the heart have been detailed in $\mathrm{Ccm}$ animal models [10-12]. Enlarged heart chambers and large blood vessel changes have been described in Ccml (santa, san) and $\mathrm{Ccm} 2$ (valentine, vtn) mutant zebrafish lines, respectively [10-13]. It has been postulated that both $\mathrm{Ccm} 1$ and $\mathrm{Ccm} 2$ regulate the concentric growth of myocardium without changing the fate of cardiomyocytes or endothelial cells [12]. In mice, $\mathrm{Ccm} 1$ knockout has been reported to induce dilation of heart chambers and large arteries (such as the aorta) and to preferentially narrow certain arteries, leading to the hypothesis that $\mathrm{Ccm} 1$ is essential for vasculogenesis, the de novo formation of the blood vessel backbone. Unlike previous zebrafish studies, associated heart defects in mice have been presumed secondary to vascular malformation [14]. Two recent studies in zebrafish embryos posit that $\mathrm{Ccm} 1$ and $\mathrm{Ccm} 2$ mutations cause the dilation of major vessels (especially primitive veins) and the progressive thinning of endothelial cells lining these primitive vessels, suggesting that $\mathrm{Ccm} 1$ proteins regulate VEC genesis, particularly vascular tubule formation during vasculogenesis, and supporting the previous perturbed vasculogenesis hypothesis in mouse models [13-15]. Two recent studies in mice and zebrafish further emphasized defective endothelial association and barrier function, or excessive dysfunctional sprouting which leads to vessel enlargement in the primitive vessels of Ccm2 mutants [16, 17].

This study advances prior research on CCM in several ways. First, by studying san zebrafish lacking $\mathrm{Ccm} 1$, we have established a critical role for $\mathrm{Ccm} 1$ in the promotion of angiogenesis and, in contrast to prior reports, have found vasculogenesis and earlier angiogenic events to be unaffected by $\mathrm{Ccm} 1$ deficiency. Instead, $\mathrm{Ccm} 1$ plays a major role in the regulation of microvascular vacuole formation, fusion, and lumenization during angiogenesis in zebrafish embryonic development. The loss of $\mathrm{Ccm} 1$ induces defective microvascular angiogenesis by disrupting microvascular lumenization. Importantly, the defective lumenization was not caused by a loss of blood flow but by the inability of VECs to form vacuoles and hence lumens. This microvascular pathology in zebrafish mirrors the pathogenesis of human CCM lesions, in which lumenless capillaries (termed collapsed vessels) are inter- twined with lumen-enlarged capillaries (termed dilated vessels or cavities). As such, Ccm1 mutant zebrafish provides an excellent model to study the pathogenesis of CCM in humans.

Second, we have elucidated the molecular mechanisms by which CCM1 deficiency results in failed microvascular development. This pathophysiology was investigated using in vitro studies in human umbilical vein endothelial cell (HUVEC) lines. As with zebrafish, the loss of CCM1 disrupts vascular lumenization in HUVECs. The absence of CCM1 inhibits the activation of Rac-1 small G protein, a key molecule that regulates the induction of vacuole formation, fusion, and lumenization in VECs.

\section{Materials and Methods}

\section{Zebrafish Care}

Zebrafish lines used in this experiment were purchased from the Zebrafish International Resource Center (Eugene, Oreg., USA). Zebrafish maintenance was performed according to the established Kimmel protocol [18]. A double transgenic line was created by crossing a $\mathrm{Ccm} 1$ mutant strain (KRIT1 ${ }^{\text {ty } 219 \mathrm{c} /+(\mathrm{AB})}$ ) with a $\operatorname{Tg}\left(\right.$ fli1:EGFP) ${ }^{\mathrm{y} 1 /+(\mathrm{AB})}$ strain in which the robust expression of EGFP in vascular endothelium enabled us to perform sensitive time-lapse microscopic imaging of blood vessels in developing embryos in vivo. $\mathrm{Ccm} 1$ mutant $\left(\mathrm{CCM1}^{-/-}\right)$zebrafish (santa, san) with $\mathrm{Tg}$ (fli1:EGFP) background were identified by their distinctive phenotype of deficient circulation and enlarged heart chambers, starting 36-48 hpf (hours post fertilization) [10-12, 19]

\section{Imaging and Microangiography}

Embryos at 24-72 hpf were dechorionated using Watchman's forceps, anesthetized in $0.6 \%$ tricaine, and mounted in $0.5 \%$ agarose on depression slides. Fluorescent images or time-lapse videos were acquired with a Nikon 90i microscope and a Coolsnap ES CCD camera (Roper Scientific), and processed using Metamorph 6.3 (MDS). After imaging, embryos were recovered by washing with system water, and placed individually into 24 -well culture plates to allow them to develop for phenotype checking as described before [19].

\section{Morpholino Analysis}

Antisense oligonucleotide morpholinos (MOs) to Ccml gene (MO1 and MO2; GeneTools) were diluted in $1 \times$ Danieau's buffer to a $0.5-\mathrm{mm}$ concentration as previously described $[12,19]$. The injection of $\mathrm{MO}$ solution (volumes: 0.1, 0.2, 0.5, 1, and $2 \mathrm{nl}$ ) or control buffer was performed on fertilized eggs at 1-cell stage from $\mathrm{Tg}$ (fli1:EGFP) zebrafish under $\mathrm{AB}$ wild-type background. After injection, the embryos were allowed to develop to designated stages at $28.5^{\circ} \mathrm{C}$.

Whole-Mount Immunofluorescent Staining of Zebrafish

Fixed zebrafish embryos were permeabilized, blocked, and incubated with primary antibodies overnight at $4^{\circ} \mathrm{C}$. Embryos were then washed and incubated with Alexa fluor 488- or 563-conju- 
gated secondary antibodies (Invitrogen) for $1 \mathrm{~h}$, followed by washing, mounting, and imaging.

\section{Endothelial Cell Culture and RNAi}

HUVECs were cultured in EGM2 medium (Clonetics). siRNA duplex targeting human CCM1 (KRIT1) and ICAP1 $\alpha$ RNA were used as previously described [3]. All gene-specific siRNA oligos and commercially available control siRNA duplex SCRAMBLE II and luciferase GL2 duplex were synthesized at Dharmacon, and transfections were carried out with Oligofectamine reagent (Invitrogen) as described by the manufacturer.

\section{Cell Migration and Tube Formation Assay}

The cell migration assay was carried out using a multiwell insert system (BD Biosciences). In each well, 15-25 × 10 3 HUVECs were incubated at $37^{\circ} \mathrm{C}$ in the insert for $16 \mathrm{~h}$. After HUVEC migration through the pores, they were fixed and stained with DiffQuik (Behring). The endothelial cell transwell migration was counted under the microscope. After RNAi treatment, $2 \times 10^{4}$ HUVECs/well were incubated for $12 \mathrm{~h}$ at $37^{\circ} \mathrm{C}$ on ECMatrix (in vitro angiogenesis assay kit; Chemicon). Tube formation processes were imaged and analyzed using the branch point counting method (Chemicon).

\section{Antibodies, Immunocytochemistry, and Western Blot}

Primary antibodies, AK (C-19), Cdc42 (B-8), and Racl (23A8), were purchased from Upstate. For the small GTPase assay, cells were lysed, and equal amounts of lysate samples from each treatment were loaded on SDS-PAGE directly or co-immunoprecipitated with PAK-1 PBD agarose beads (cat. No. 14-325, Upstate). Protein concentrations of the RNAi treatment lysates were measured using the RC DC protein assay (Bio-Rad). Equal amounts of protein from each treatment were then loaded on SDS-PAGE and detected with designated antibodies followed by chemiluminescence (GE).

\section{Statistical Testing}

For multiple comparisons, one-way analysis of variance (ANOVA) was used to detect differences in the mean values among all treatment groups. Pairwise comparison (t test) was used to test the difference between each treatment.

\section{Results}

Vasculogenesis Is Normal in san Zebrafish Embryos

The backbones of large axial arteries and veins are formed de novo through the migration and coalescence of endothelial precursor cells from mesoderm, a process called vasculogenesis [20,21]. To observe the effect of loss of $\mathrm{Ccm} 1$ function on vasculogenesis in embryonic zebrafish, dynamic fluorescent vasculature images were acquired in vivo from wild-type and $\mathrm{Ccm} 1$ mutant $\left(\mathrm{Ccml}^{-1-}\right.$, san) embryos. At 24-26 hpf, a primitive network of axial vessels consisting of dorsal aorta (DA), posterior cardinal vein (PCV), caudal artery (CA), and caudal vein
(CV) were observed. From $24 \mathrm{hpf}$, DA and PCV form distinct lumens and start circulating primitive blood along primitive vessels as they function as shunt. In contrast to previous reports $[13,15]$, we observed no significant differences in vasculogenesis between wild-type and san zebrafish embryos. Additionally, the distribution of vasculature, lumenization of large arteries and veins, and aortic structures were comparable in all embryos (fig. 1; online suppl. fig. 1, video 1; for all online supplementary material, see www.karger.com/doi/10.1159/000316851), affirming that $\mathrm{Ccm} 1$ is not required for the primitive axial vessel formation of vasculogenesis.

\section{Sprouting in Primary Angiogenesis Is Unaffected in san Zebrafish Embryos}

Two distinct angiogenic events have been temporally defined during embryonic angiogenesis: a primary artery-driven vascular lattice first forms in primary angiogenesis, followed by the emergence and growth of a network of vein-driven sprouts in secondary angiogenesis $[20,21]$. The emergence of primary arterial sprouts begins at $20 \mathrm{hpf}$, a few hours before a patent axial plexus is fully developed (23-24 hpf), and usually spans from 20 to 36 hpf. These sprouts are originated from primitive arteries (DA and CA), and they form rudimentary intersegmental arteries (SeA); SeA will ultimately migrate dorsally, and connect with each other to form new dorsal longitudinal anastomotic vessels (DLAV). The vast majority of SeAs will remain as lumenless cords of VECs until secondary angiogenesis begins.

Our observation indicated that the sprouting of VECs from large arteries (DA and CA) appeared the same in san and wild-type embryos at $24 \mathrm{hpf}$. At this stage, no lumens were established in the newly established SeA. However, rhythmic beatings of primitive heart tubes and slow blood flow within the large primitive vessels (DA, $\mathrm{CA}, \mathrm{PCV}$, and $\mathrm{CV}$ ) were observed in all wild-type embryos and $\mathrm{Ccm} 1$ mutants, as indicated by the vigorous contraction of GFP-illuminated endocardium. We observed no difference in the beating frequency and lumen diameter of the heart, and SeA lattice formation in wildtype and san embryos at this embryonic stage (fig. 1; online suppl. fig. 1, video 1).

\section{Sprouting in Secondary Angiogenesis Is Uninterrupted} in san Zebrafish Embryos

As primary embryonic angiogenesis concludes, secondary angiogenic vessel sprouting starts immediately, lasting from 36 to $72 \mathrm{hpf}$. Secondary sprouts emerge exclusively from large veins (PCV and CV) to form inter- 
Fig. 1. Early vasculogenesis and angiogenesis at $24 \mathrm{hpf}$. Lateral views of primitive vessels with $\mathrm{Tg}$ (fli1:EGFP) background in both wild-type and $\mathrm{Ccm} 1$ mutant embryos at $24 \mathrm{hpf}$. In the aortic area, morphology and lumens of lateral DA (LDA), middle brain vein $(\mathrm{MCeV})$, primordial hindbrain channel (PHBC), and primordial midbrain channel (PMBC) appeared comparable in both wild-type and Ccml mutant embryos (a, b, marked by arrows, respectively). Note that no obvious abnormal primitive vessels were identified in either wild-type or Ccm 1 mutant embryos. VECs gradually sprout from the primitive arteries (DA and CA) in a rostral-caudal direction, forming pairs of rudimentary $\mathrm{SeA}$ (c, d). VECs in SeA migrate dorsally and then ramify laterally, forming $\operatorname{DLAV}(\mathbf{c}, \mathbf{d})$. At this stage, SeA and DLAV belong to a primary angiogenic network. (Online version: red and blue arrows point to the blood flow direction in the DA and PCV, respectively; c, d.) Of note, only lumenless vessel cords are formed in the SeA and DLAV of both wild-type and Ccml mutant embryos. Bars $=100 \mu \mathrm{m}$.

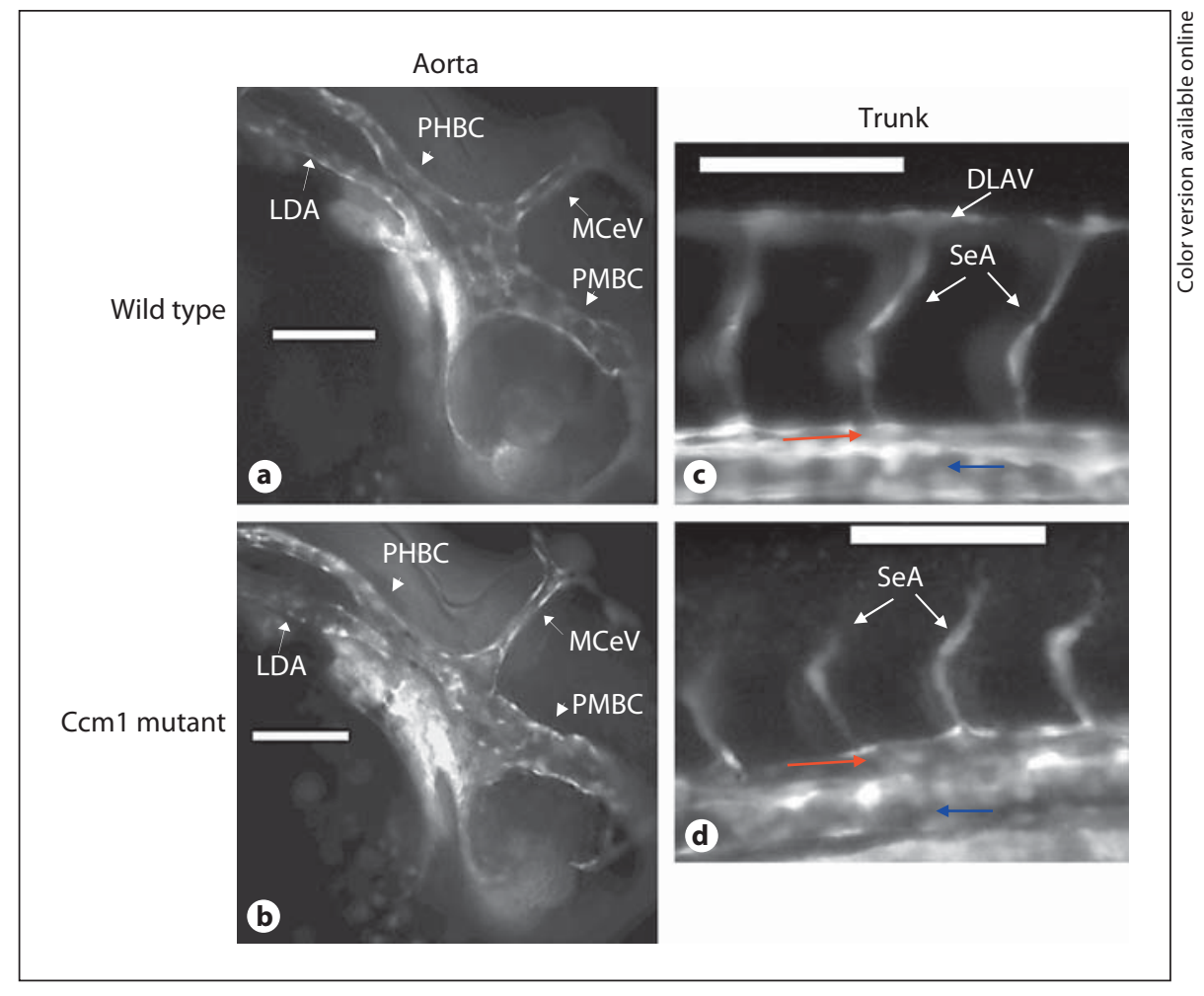

segmental veins ( $\mathrm{SeV})$. Half of SeVs make stochastic connections to the adjacent primary segments (SeAs), linking the primitive veins ( $\mathrm{PCV}$ and $\mathrm{CV}$ ) to the primary angiogenic vascular network. The remaining half of SeVs runs dorsally to form the parachordal vessel in the middle of vessel networks. The combination of a SeA and a $\mathrm{SeV}$ is called an intersegmental vessel (ISV). By the end of this embryonic stage (72 hpf), two completed angiogenic ISV networks have been formed on each side of the trunk, and stochastically connected SeAs and SeVs have been transformed from cord-like lattices to a fully functioning network of patent vessels [21].

Imaging revealed that the sprouting of VECs from PCV and CV appeared the same in both san and wildtype embryos throughout the secondary angiogenesis. The formation of the zebrafish secondary vessel networks was identical in $\mathrm{Ccm} 1$ mutants and their wild-type siblings at $36 \mathrm{hpf}$ (fig. 2a-d), $48 \mathrm{hpf}$ (fig. 2e-h), and $72 \mathrm{hpf}$ (fig. $2 \mathrm{i}$, j; online suppl. fig. 1 , video 2 ).

\section{Microvascular Lumenization Is Perturbed in san Zebrafish Embryos}

While the development of the microvascular network was similar in wild-type and san embryos through $72 \mathrm{hpf}$, the formation of lumens was distinctly different in these two lines. By screening newly developed SeAs right at the end of primary angiogenesis in wild-type and san embryos, we found that, in wild-type zebrafish, all SeAs showed some degree of early lumenization, but only a small percentage of SeAs ( $7.6 \pm 2.4 \% ; n=65$ SeAs) fully developed continuously open lumens. The lumenization of SeA started rostrally and proceeded caudally along the vessel plexus. In contrast, no lumen formation was found in SeAs of $\mathrm{Ccm} 1$ mutants at the same developmental stage $(n=80$ SeAs). The difference in lumen formation at the end of primary angiogenesis is statistically significant ( $p<0.05$, $t$ test). Considering the fact that the hemodynamics in primitive vessels at this stage were quite comparable between wild-type and $\mathrm{Ccm} 1$ mutant (online suppl. video 1), we concluded that Ccml deficiency results in failed lumenization in SeAs during the primary angiogenesis.

From $36 \mathrm{hpf}$, we observed that the sprouting SeVs started to connect with primary SeAs to form an ISV network in a relatively rapid manner: ISV lumenization and subsequent initiation of circulation started momentarily after $\mathrm{SeV}$ s developed (fig. 2b). At this stage, angiogenic vessels, including all ISVs and cranial microvasculature, 


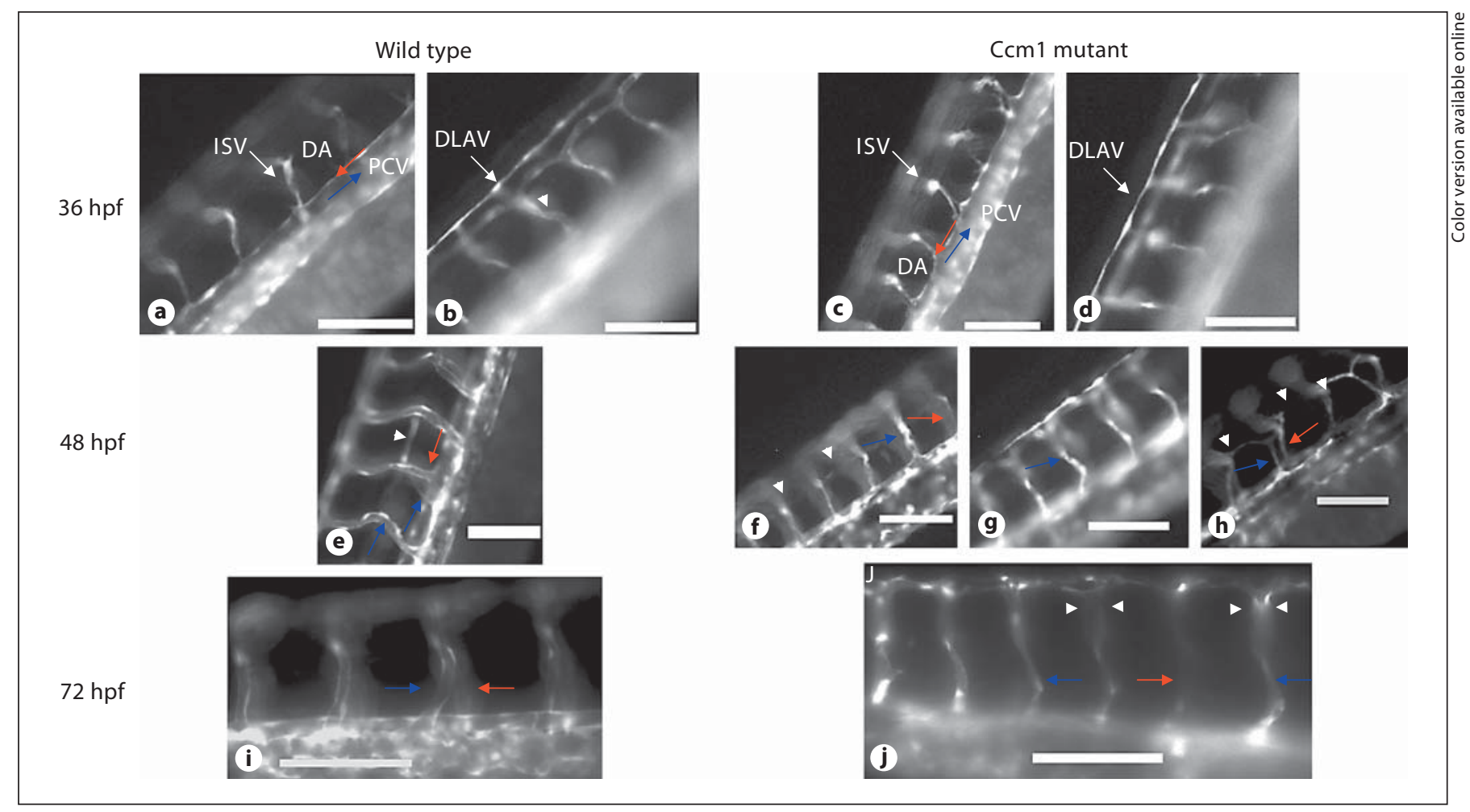

Fig. 2. Microvasculature morphogenesis from 36 to $72 \mathrm{hpf}$. Lateral views of vasculatures with $\mathrm{Tg}$ (fli1:EGFP) background in wildtype $(\mathbf{a}, \mathbf{b}, \mathbf{e}, \mathbf{i})$ and $\mathrm{Ccm} 1$ mutant embryos $(\mathbf{c}, \mathbf{d}, \mathbf{f}, \mathbf{g}, \mathbf{h}, \mathbf{j})$ at 36,48 , and $72 \mathrm{hpf}$, respectively. At $36 \mathrm{hpf}$, VECs begin to sprout from PCV to form SeVs and connect with SeAs, forming ISVs, marked by white arrows, both of which are still lumenless cords (a, c). (Online version: red and blue arrows point to the blood flow direction in the DA and PCV, respectively.) $\mathbf{b}, \mathbf{d}$ Parallel z-scans of embryos in $\mathbf{a}$ and $\mathbf{c}$, respectively. In these sections, the initiation of ISV lumen formation can be noted in the wild-type embryo (arrowhead; b) but not in Ccml mutant (d). At $48 \mathrm{hpf}$, lattices of pri- mary SeA (online version: red arrows) and secondary SeV (online version: blue arrows) connect with each other to form the normal ISV network in both wild-type and mutant embryos $(\mathbf{e}, \mathbf{f}, \mathbf{g}, \mathbf{h})$. Secondary ISV sprouts from PCV also form parachordal vessel, marked by arrowheads (e, f, h). At $72 \mathrm{hpf}$, normal blood circulation between SeA (online version: red arrows) and SeV (online version: blue arrows) was demonstrated in wild-type embryos (i), but not in Ccml mutant embryos (j). The dorsal portion of ISVs developed noticeable funnel-like enlargements marked by arrowheads in Ccml mutant embryos (j). Bars $=100 \mu \mathrm{m}$.

arrowhead), a vessel phenotype identical to the zebrafish mutant Silent heart (sih). The ventral ends and central sections of the ISVs remained closed cords at $48-72 \mathrm{hpf}$ (fig. $2 \mathrm{f}, \mathrm{h}, \mathrm{j}$ ). These findings indicate that the angiogenic lumen formation during angiogenesis is ceased in san embryos (fig. 2, 3; online suppl. fig. 1).

\section{Early Circulation through the Heart and Primitive \\ Vessels Is Unaffected in san Zebrafish Embryos \\ Circulation in Large Primitive Vessels}

The earliest circulation of blood cells within primitive vessels (DA, CA, CV, and PCV) starts at 24-26 hpf, followed by circulation in a rostral loop in the head [20]. Previous research has observed the cessation of circulation through large primitive vessels (DA, PCV, CA, and 


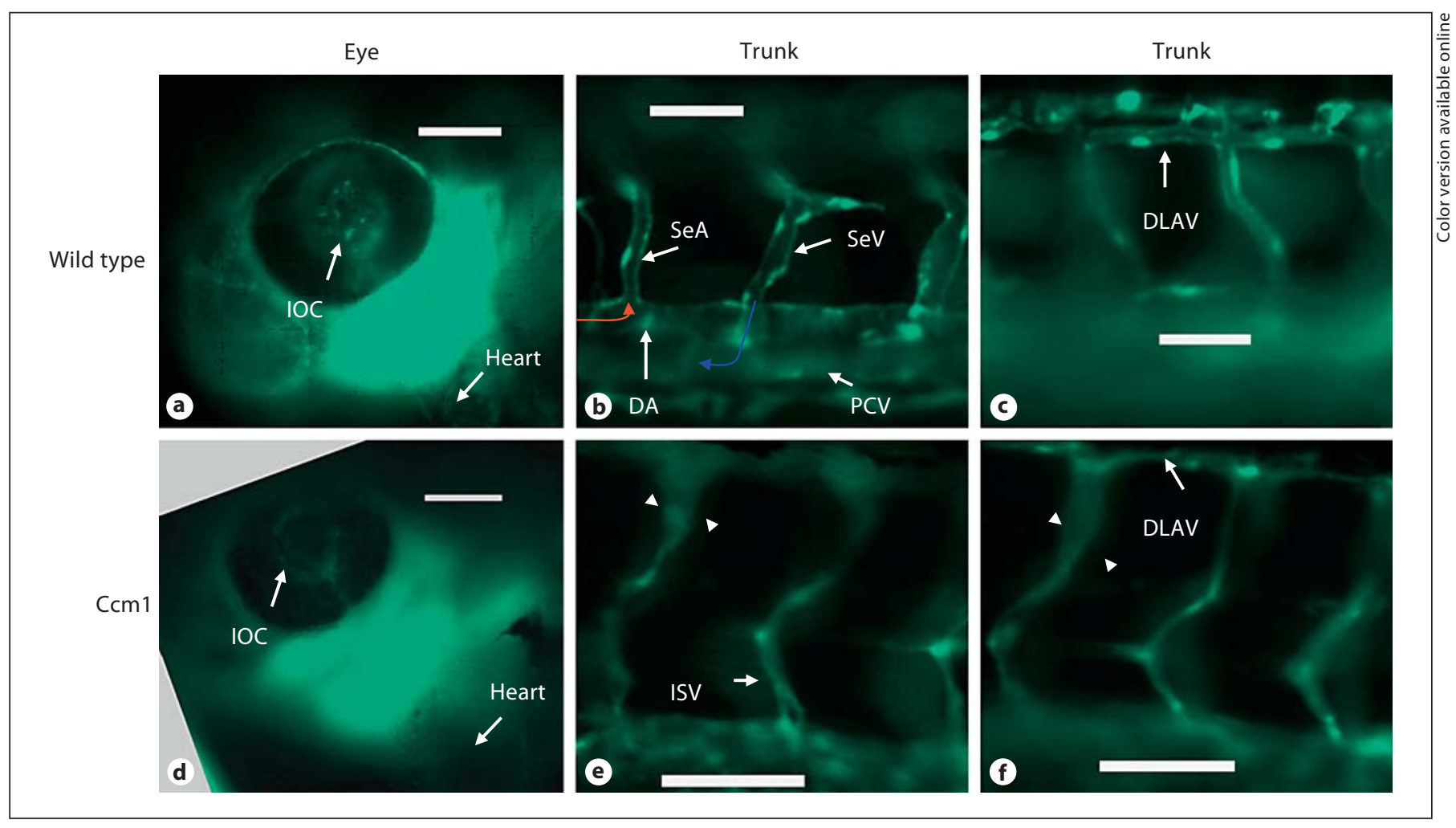

Fig. 3. Detailed vascular pathogenesis of $\mathrm{Ccm} 1$ mutant embryos at $48 \mathrm{hpf}$. Further examination of the EGFP-labeled microvasculature in $48 \mathrm{hpf}$ embryos identifies angiogenic microvessels located at the head and trunk-tail areas, such as the inner optical circle (IOC), SeA and SeV, and DLAV. Those microvessels develop normal lumen and interconnections in the wild type (a-c), but they are collapsed without normal lumen in Ccm 1 mutants (d-f). (Online version: red and blue arrows show the direction of blood flow in artery and vein, respectively; b.) The dorsal funnel-like enlargement in ISV is marked by arrowheads in Ccml mutant embryos (f). Bars $=50 \mu \mathrm{m}$.

Defective Microcirculation Followed by

Cardiovascular Malformations in san Zebrafish

At $28 \mathrm{hpf}$, the heart of san embryos starts to show signs of defective blood flow [12]. We observed that in san embryos, the flow of blood cells became stagnant within CCV albeit vigorous heart contractions, indicating the initially damaged atrium-ventricle structure. From $32 \mathrm{hpf}$ and beyond, the defective circulation was accompanied with prominently dilated heart and progressive lumen malformation in primitive vessels, as arteries (DA and CA) started to narrow while PCVs started to dilate in $\mathrm{Ccm} 1$ mutant embryos. CVs remained as large venous sinus in $\mathrm{Ccm} 1$ mutant embryos, while those in wild-type embryos developed into venous plexuses consisting of interconnecting veins with increasing bright EGFP signals. Both the magnitude of heartbeat and the size of the heart chamber of san embryos increased significantly at $36 \mathrm{hpf}$, indicating the continuation of cardiac dilatation and atrium-ventricle

J Vasc Res 2011;48:130-140 
Fig. 4. The hollow cavity generated by vacuole formation in VECs eventually leads to lumen formation in the microvasculature. At $28 \mathrm{hpf}$, many developing vacuoles can be found in newly sprouting SeA during primary angiogenesis in the trunk of wildtype embryos (a, white arrows), but not in Ccm1 mutants (d). Well-developed and enlarged vacuoles in VECs in ISVs progressively establish large hollow cavities (white arrows) through the coalescence of vesicles called vacuoles in both SeAs and $\mathrm{SeVs}$ in wild-type embryos during secondary angiogenesis (b). These cavities lead to lumen formation before the blood circulation is established at $36 \mathrm{hpf}$ (c, white arrows). In contrast, ISVs in Ccm 1 mutant embryos do not have these hollow cavities, but remain as lumenless vessels (e). Bars = $50 \mu \mathrm{m}$.

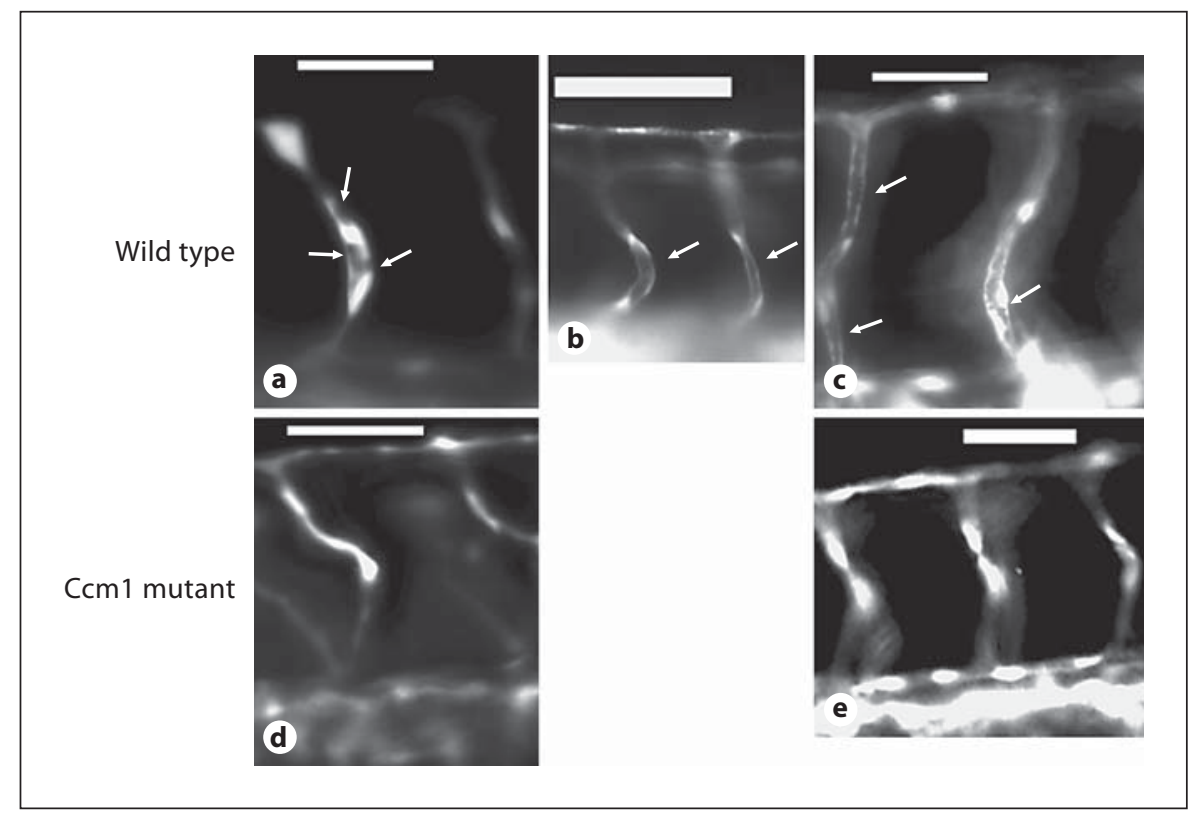

structural destruction. By $48 \mathrm{hpf}$, the heart chamber of san embryos was dilated tremendously and the atrium-ventricle structure was disrupted. Blood cells had accumulated within heart chambers and large vessels adjacent to the heart such as the common cardinal vein and DA. Consequently, no effective circulation of blood cells was observed in the large vessels from this point on. Microangiography studies at $48 \mathrm{hpf}$ confirmed the absence of blood flow in both large primitive vessels and small cranial vessels of san embryos compared with normal blood flow in those vessels in wild-type embryos (online suppl. fig. 2).

\section{Failed Lumen Formation Is due to Dysregulated}

Vacuole Formation in VECs in san Zebrafish Embryos

Both in vitro and in vivo studies have proven that intracellular vacuole formation is required for VEC lumen formation [22-24]. These vacuoles coalesce and establish large intracellular and/or intercellular hollow cavities, eventually leading to vascular lumen formation [23-25]. In in vitro angiogenesis assay, VECs form cords of invading cells during angiogenesis. Vacuoles have been observed in VECs leading the invasion front, indicating vacuole formation might contribute to the directional expansion of VEC tube networks to facilitate both sprouting and lumen formation [26-28].

During angiogenesis in wild-type embryos, imaging uncovered that vacuoles were visible in VECs as they contributed to the process of lumen formation. These vacu- oles were not visible in the VECs of san embryos. Specifically, upon examination of VECs during primary VEC sprouting at $28 \mathrm{hpf}$, primitive vacuoles were observed in VECs in wild-type embryos (fig. 4a) but not in san zebrafish (fig. $4 \mathrm{~d}$ ). At $36 \mathrm{hpf}$ during secondary angiogenic sprouting, intracellular vacuoles significantly matured and clustered within VECs along ISV (fig. 4b) and eventually formed intracellular and/or extracellular hollow cavities in wild-type embryos (fig. 4c) but not in $\mathrm{Ccm} 1$ mutants (fig. 4e). These findings support the role of $\mathrm{Ccm} 1$ in the vacuolization of VECs prior to microvascular lumenization. As such, $\mathrm{Ccm} 1$ is a key regulator modulating lumenization during angiogenesis.

\section{MO Knockdown of Ccm1 Protein Disrupts Lumen \\ Formation in Zebrafish Embryos}

To further investigate the relationship of $\mathrm{Ccm} 1$ deficiency to failed lumenization, we performed a dose-phenotype study of Ccm1 MO knockdown and analyzed its effect on microvascular structure and circulation. It has been known that Sih zebrafish feature a lack of circulation which secondarily induces constriction of large vessels, which might lead to defective lumenization of microvasculature [21]. Considering the presence of defective microvascular lumenization in both san and sih zebrafish lines, the possibility exists that the same mechanism responsible for failed lumenization in sih zebrafish (i.e. a primary lack of circulation) also occurs in san zebrafish. 

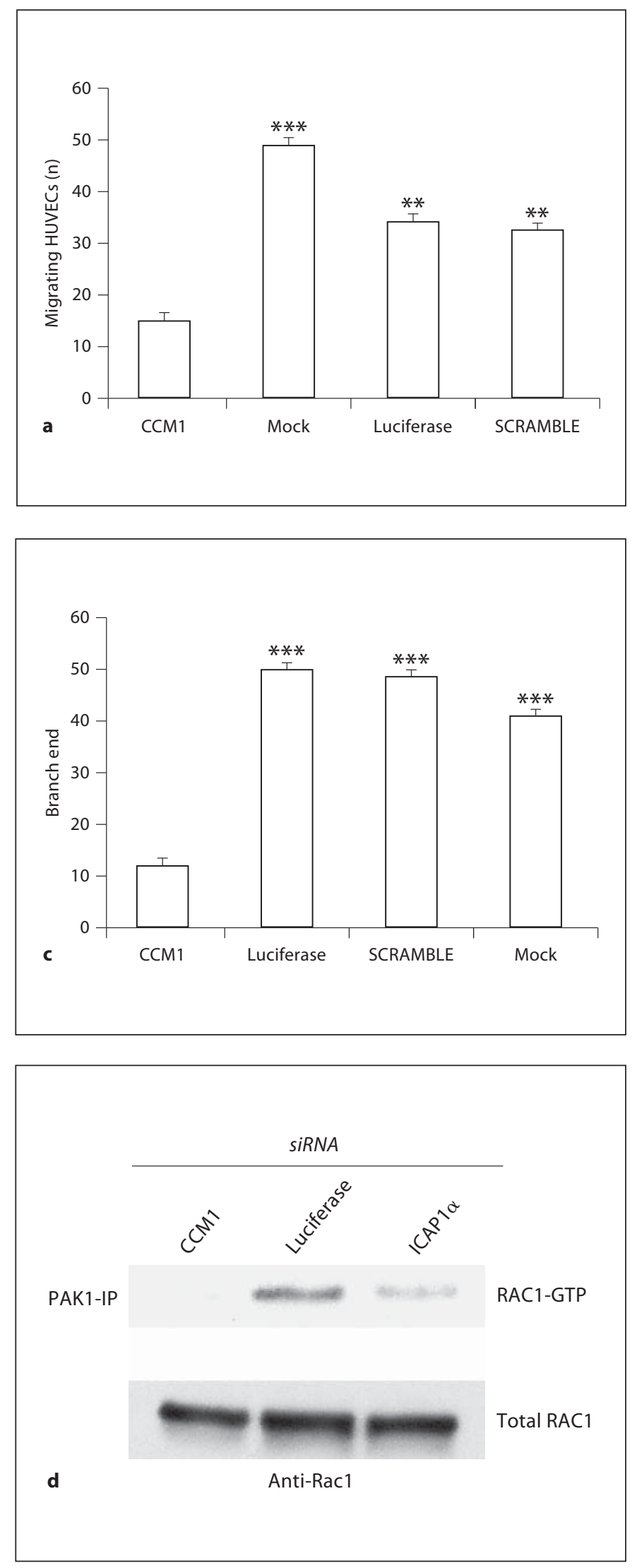

CCM1 Modulates Microvascular Vacuole and Lumen Formation

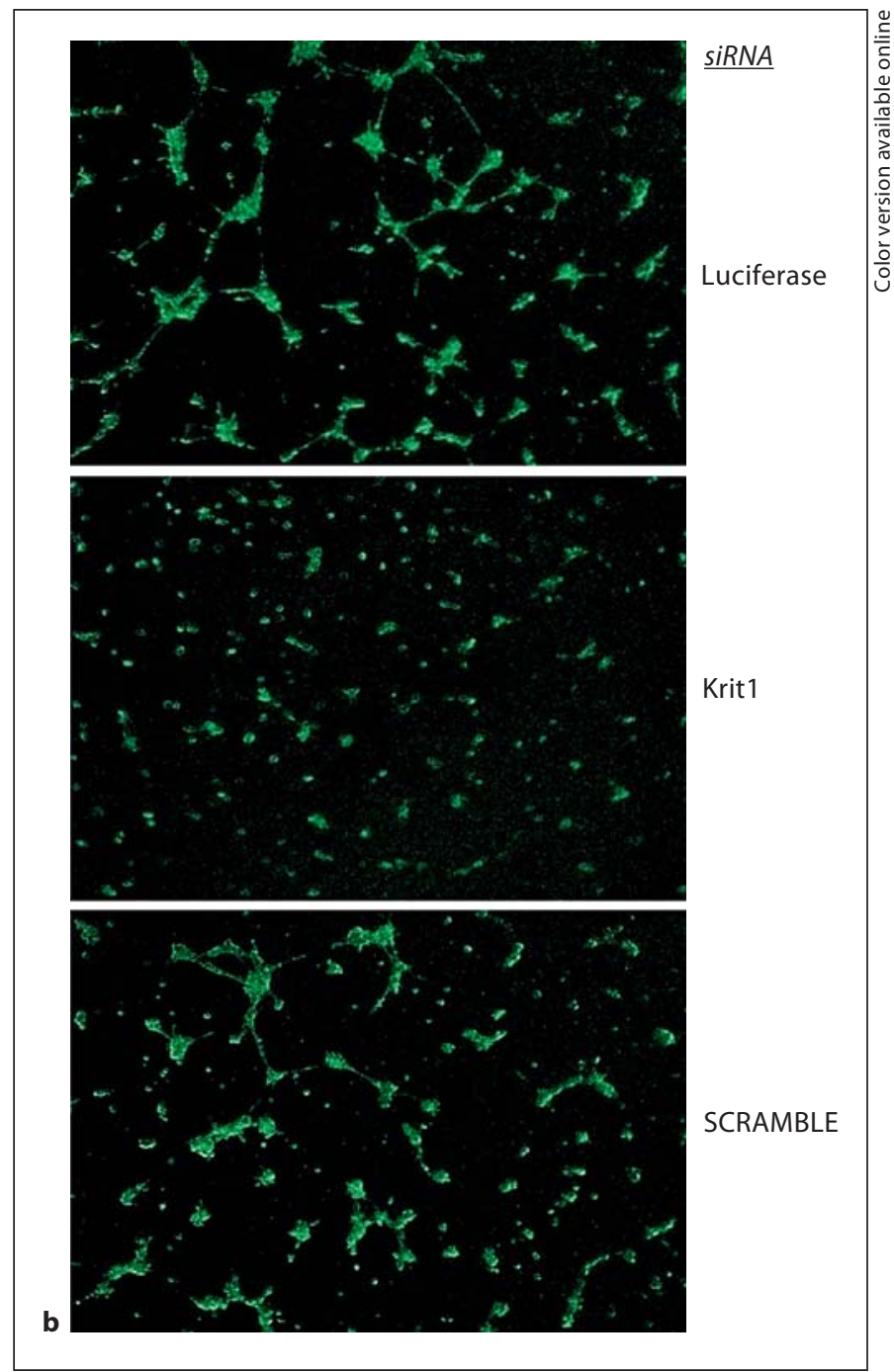

Fig. 5. Depletion of CCM1 and ICAP1 $\alpha$ leads to decreased HUVEC angiogenesis. a The number of migrating HUVECs treated with CCM1 siRNA was significantly decreased compared to siRNA controls (one-way ANOVA, F $=14.48$, *** $\mathrm{p}<0.001$, $\mathrm{n}=4$ ). Pairwise comparisons (t test) show significant differences between CCM1 siRNA and each of the controls ${ }^{* *} p \leq$ 0.001). b Angiogenesis is severely impaired due to silencing of CCM1. c The branch point counting method was used for quantification (one-way ANOVA: $\mathrm{F}=35.99,{ }^{* * *} \mathrm{p}<0.001$ ). d Western blot analysis revealed decreases in GTP-bound Racl (the active form of Rac1) with CCM1 and ICAP1 $\alpha$ depletion compared to the luciferase siRNA-treated control. Total Racl is shown as a loading control.

J Vasc Res 2011;48:130-140 
Table 1. Dose-dependent inhibition of vascular performance and circulation by $\mathrm{Ccm} 1 \mathrm{MOs}$ at $48 \mathrm{hpf}$

\begin{tabular}{|c|c|c|c|c|}
\hline Treatment & $\begin{array}{l}\text { Volume } \\
\text { injected } \\
\mathrm{nl}\end{array}$ & $\begin{array}{l}\text { Normal } \\
\%(\mathrm{n})\end{array}$ & $\begin{array}{l}\text { Loss of ISV } \\
\text { circulation } \\
\text { only, \% (n) }\end{array}$ & $\begin{array}{l}\text { Total loss of } \\
\text { circulation \& } \\
\text { enlarged heart, \% (n) }\end{array}$ \\
\hline \multirow[t]{4}{*}{ Ccm 1 MO-1 } & $0.1-0.2$ & $45.2(14)$ & $38.7(12)$ & $16.1(5)$ \\
\hline & 0.5 & $25.0(4)$ & $37.5(6)$ & $37.5(6)$ \\
\hline & 1.0 & $8.4(2)$ & $20.8(5)$ & 70.8 (17) \\
\hline & 2.0 & $6.7(2)$ & $10.0(3)$ & $83.3(25)$ \\
\hline Control MO & 2.0 & $100(28)$ & 0 & 0 \\
\hline
\end{tabular}

The distribution of three phenotypes (normal, loss of ISV circulation only, and total loss of circulation and enlarged heart) varies among different dosages of $\mathrm{Ccm} 1 \mathrm{MO}$ treatment at $48 \mathrm{hpf}$.

A gradient of $\mathrm{Ccm} 1 \mathrm{MO}$ was injected into wild-type embryos. After secondary angiogenesis at $48 \mathrm{hpf}$, phenotypes of Ccm1 MO zebrafish vascular systems were classified into three categories (online suppl. fig. 3). Phenotype 1 (normal perfusion with patent vasculature) was detected in wild-type embryos. Phenotype 2 (loss of ISV circulation but normal circulation in major primitive vessels, e.g. DA, PCV, CA and CV; online suppl. video 3) was noted in wild-type embryos before $36 \mathrm{hpf}$ or san embryos but not in sih embryos. For this MO phenotype, ISV circulation appeared in all-or-none fashion, no partial phenotype 2 (in which ISV circulation appears normal at the rostral end but gradually deteriorates caudally along the vessel plexus until no circulation in ISV can be seen) could be identified. Phenotype 3 (total loss of circulation with an enlarged heart) was found to be identical to the vascular condition of the san zebrafish at $48 \mathrm{hpf}$ and beyond.

At low MO dosages (0.1-0.2 nl), approximately half of the san embryos had phenotype 1, approximately half exhibited phenotype 2 , and a small percentage showed phenotype 3 . At medium dosage $(0.5 \mathrm{nl})$, the san embryos were evenly distributed among the three phenotypes. At high dosages (1-2 nl), the majority of the san embryos exhibited phenotype 3 (table 1).

The phenotypes outlined above, especially the newly defined phenotype 2 , indicated that $\mathrm{Ccm} 1$ is essential for the lumenization of ISVs and hence the successful establishment of circulation during secondary angiogenesis. With the role of $\mathrm{Ccm} 1$ established in zebrafish, we next turned our focus to understanding the mechanisms by which CCM1 promotes lumenization and by which CCM1 absence causes failed lumenization in human endothelial cells.

\section{Depletion of CCM1 Results in Failure to Form VEC}

Tubes and Decreased Cell Mobility

Silencing of CCM1 Impairs Integrin-Modulated Cell Mobility

In addition to vacuole formation, VEC angiogenesis requires endothelial cell proliferation, mobility, and survival, for example. We undertook several in vitro studies using cultured HUVECs to assess whether CCM1 deficiency affects any of these angiogenic variables. We found that silencing of CCM1 with RNAi significantly inhibits endothelial HUVEC migration capacity (fig. 5a), indicating silencing of CCM1 in VECs has a significantly negative impact on cell mobility.

In in vitro angiogenesis assays, we found that the impairment in angiogenesis can be observed visually (fig. 5b) and quantified (fig. 5c). It should be noted that our CCM1 RNAi in vitro tube formation assay results are consistent with Cdc42 and Racl RNAi data [27], indicating that CCM1 might share the same signaling pathway with Cdc42 and Racl small GTPases which are essential regulators in VEC mobility and vacuole formation.

\section{Silencing of CCM1 Inhibits Racl GTPase Activity}

Cdc42 and Racl GTPases have been proven to be required for vacuole and lumen formation $[23,26,27]$. ICAP1 $\alpha$ has been reported to interact with $\mathrm{Cdc} 42$ and Racl, and modulates the activity of these GTPases [28]. The CCM complex not only regulates Racl activity through ICAP1 $\alpha$ but also directly interacts with Rac1 $[1,3,9,28]$.

With in vitro experimentation, silencing of either CCM1 or its cellular effector, ICAP1 $\alpha$, significantly decreases steady-state abundance of Racl-GTP, a known positive regulator of cell motility and a key modulator of vacuole and lumen formation (fig. $5 \mathrm{~d}$ ). No obvious changes in Cdc42 activity by silencing CCM1 were observed (data not shown). In combination with previous findings that the CCM complex not only regulates Racl activity through ICAP $1 \alpha$ but also directly interacts with Rac1 [1, $3,9,28]$, our data provide strong evidence that CCM1 is upstream of Racl small GTPase and regulates Racl activity through ICAP $1 \alpha$ in the angiogenic signaling pathway during vacuole and lumen formation.

\section{Discussion}

Using both in vivo animal models and in vitro human VEC lines, we have demonstrated that $\mathrm{Ccm} 1$ deficiency causes failed vacuole and lumen formation in angiogenic ISVs through Rac-1 small GTPase signaling. We have also 
determined that previously reported major cardiovascular defects in Ccml zebrafish might be secondary to microvascular pathogenesis. This study has established a paradigm that links $\mathrm{Ccm} 1$ molecular function to VEC morphogenesis during microvascular angiogenesis.

The morphology changes of large vessels and the heart in san embryos are very possibly caused by increases in contractile pressure in vein and heart but a decrease in arteries. The enlargement in the heart, caused by either failed concentric growth or increases in blood pressure, disturbs atria-ventricle valve function and results in back-feeding of the circulation to the vein. These overall morphological changes deteriorate progressively with time because of the law of Laplace: The larger the vessel radius, the larger the wall tension required to withstand a given internal fluid pressure.

One can argue that the circulation in Ccml mutant zebrafish embryos may appear normal during earlier embryonic stages, the inability of the heart in these animals to generate normal pressures may prevent the flow through small-diameter microvessels which could influence lumenization, as occurs in sih zebrafish. Although the obvious cardiac phenotype may not be apparent until $32 \mathrm{hpf}$, the functional consequences (i.e. defective pressure generation due to cardiac insufficiency) will manifest much earlier. This argument might be solid for the cause of defective ISV lumenization during secondary angiogenesis when circulation is dramatically decreased in Ccml zebrafish; however, it could not challenge the observation of the total loss of ISV lumenization during primary angiogenesis in san embryos. Furthermore, in normal embryos, the embryonic development of the cardiovasculature takes place gradually with time, and angiogenesis (and lumenization) of ISV occur not only gradually with time, but also sequentially from rostral to caudal along major primary blood vessels. Therefore in san embryos, if the underlying pathology is primarily caused by lack of perfusion due to defective cardiac growth and pump dysfunction, the failed lumenization of ISV would appear worse toward the caudal end. This is contrary to what we have observed in both Ccm1 mutant and morphant embryos. Actually, the angiogenic lumenization can only be identified in two categories: all or none. Finally, a specific phenotype (phenotype 2) generated from $\mathrm{Ccm} 1 \mathrm{MO}$ embryos shows apparently normal circulation in the large primitive vessels while the ISV circulation is disrupted in an all-or-none fashion. This phenotype further indicates that loss of ISV circulation is not caused solely by potentially changed hemodynamics originating from the heart. Also, after secondary angiogenesis (48 hpf), this phenotype mirrors the wild-type zebrafish embryos prior to the initiation of secondary angiogenesis ( $36 \mathrm{hpf}$ ) and provides evidence that ISV lumenization, a major event of secondary angiogenesis, does not occur in these MO embryos. Therefore, the presence of this phenotype in Ccml morphants indicates that the defective lumenization seen in $\mathrm{Ccm} 1$ deficiency is independent of circulation changes. In combining defective lumenization during angiogenesis and vacuole formation observation in san embryos, and $\mathrm{Ccm} 1 \mathrm{MO}$ phenotypes, our data lead to the conclusion that the hemodynamic changes in the circulation system in san zebrafish might be the consequence and not the cause of the failed lumenization.

Prior research emphasized the role of $\mathrm{Ccm}$ in vasculogenesis and VEC contacts. However, our research has found that the vital function of $\mathrm{Ccm} 1$ is angiogenesis, and perturbed angiogenesis is the origin of circulatory failure in $\mathrm{Ccm} 1$ deficiency. Specifically, $\mathrm{Ccm} 1$ promotes tubulogenesis via the regulation of microvascular lumenization. We demonstrated that defective lumenization might not be caused by a loss of blood flow, but by failure of VEC vacuole and lumen formation. We also provided in vitro evidence that CCM1 deficiency disrupts angiogenic migration and tubulogenesis in cultured HUVECs and inhibits the activation of Rac-1 small GTPase, a key molecule that regulates the induction of vacuole and lumen formation in VECs. It should be noted that secondary angiogenesis in zebrafish starts at $36 \mathrm{hpf}$. Not coincidently, expression data have demonstrated that $\mathrm{Ccm} 1 \mathrm{mRNA}$ expression occurs in the axial PCV from 28 to $48 \mathrm{hpf}$ in zebrafish embryos [12], affirming the essential role of Ccm 1 in tubulogenesis.

The implications of the similarity between san zebrafish and human CCM microvascular pathology may extend beyond pathogenetic research. We have shown that $\mathrm{Ccml}$ is a novel and essential angiogenic factor in vertebrate embryonic angiogenesis. Perhaps the most intriguing potential implication of our findings relates to cancer therapy, with the possibility that $\mathrm{Ccm} 1$ could serve as a candidate in the future development of angiogenesis-related pharmaceuticals.

\section{Acknowledgments}

We thank Chris Carr and Betty Chen (Department of Neurosurgery, University of Mississippi Medical Center), and Hal Dietz, Richard Clatterbuck, and Sharmila Basu (Johns Hopkins University) for their invaluable technical help and discussions during the preparation of the manuscript. J.Z. was supported by TTUHSC preliminary data and PLF_SOM Seed and NINDS/NIH grants. 


\section{References}

1 Zhang J, Clatterbuck RE, Rigamonti D, Chang DD, Dietz HC: Interaction between krit1 and icaplalpha infers perturbation of integrin beta1-mediated angiogenesis in the pathogenesis of cerebral cavernous malformation. Hum Mol Genet 2001;10:2953-2960.

-2 Zawistowski JS, Serebriiskii IG, Lee MF, Golemis EA, Marchuk DA: KRIT1 association with the integrin-binding protein ICAP-1: a new direction in the elucidation of cerebral cavernous malformations (CCM1) pathogenesis. Hum Mol Genet 2002;11:389-396.

-3 Zhang J, Basu S, Rigamonti D, Dietz HC, Clatterbuck RE: Krit1 modulates beta 1-integrin-mediated endothelial cell proliferation. Neurosurgery 2008;63:571-578, discussion 578 .

-4 Hilder TL, Malone MH, Bencharit S, Colicelli J, Haystead TA, Johnson GL, Wu CC: Proteomic identification of the cerebral cavernous malformation signaling complex. J Proteome Res 2007;6:4343-4355.

5 Voss K, Stahl S, Schleider E, Ullrich S, Nickel J, Mueller TD, Felbor U: CCM3 interacts with CCM2 indicating common pathogenesis for cerebral cavernous malformations. Neurogenetics 2007;8:249-256.

-6 Zawistowski JS, Stalheim L, Uhlik MT, Abell AN, Ancrile BB, Johnson GL, Marchuk DA: CCM1 and CCM2 protein interactions in cell signaling: implications for cerebral cavernous malformations pathogenesis. Hum Mol Genet 2005; 14:2521-2531.

-7 Zhang J, Rigamonti D, Dietz HC, Clatterbuck RE: Interaction between kritl and malcavernin: implications for the pathogenesis of cerebral cavernous malformations. Neurosurgery 2007;60:353-359, discussion 359.

$\checkmark 8 \mathrm{MaX}$, Zhao H, Shan J, Long F, Chen Y, Zhang Y, Han X, Ma D: PDCD10 interacts with Ste20-related kinase MST4 to promote cell growth and transformation via modulation of the ERK pathway. Mol Biol Cell 2007;18: 1965-1978.

9 Uhlik MT, Abell AN, Johnson NL, Sun W, Cuevas BD, Lobel-Rice KE, Horne EA, Dell'Acqua ML, Johnson GL: Rac-MEKK3MKK3 scaffolding for p38 MAPK activation during hyperosmotic shock. Nat Cell Biol 2003;5:1104-1110.
10 Chen JN, Haffter P, Odenthal J, Vogelsang E, Brand M, van Eeden FJ, Furutani-Seiki M, Granato M, Hammerschmidt M, Heisenberg CP, Jiang YJ, Kane DA, Kelsh RN, Mullins MC, Nusslein-Volhard C: Mutations affecting the cardiovascular system and other internal organs in zebrafish. Development 1996;123:293-302.

11 Stainier DY, Fouquet B, Chen JN, Warren KS, Weinstein BM, Meiler SE, Mohideen MA, Neuhauss SC, Solnica-Krezel L, Schier AF, Zwartkruis F, Stemple DL, Malicki J, Driever W, Fishman MC: Mutations affecting the formation and function of the cardiovascular system in the zebrafish embryo. Development 1996;123:285-292.

-12 Mably JD, Chuang LP, Serluca FC, Mohideen M-APK, Chen J-N, Fishman MC: santa and valentine pattern concentric growth of cardiac myocardium in the zebrafish. Development 2006;133:3139-3146.

13 Hogan BM, Bussmann J, Wolburg H, Schulte-Merker S: $c c m 1$ cell autonomously regulates endothelial cellular morphogenesis and vascular tubulogenesis in zebrafish. Hum Mol Genet 2008; 17:2424-2432.

14 Whitehead KJ, Plummer NW, Adams JA Marchuk DA, Li DY: $C \mathrm{~cm} 1$ is required for arterial morphogenesis: implications for the etiology of human cavernous malformations. Development 2004;131:1437-1448.

-15 Jin S-W, Herzog W, Santoro MM, Mitchell TS, Frantsve J, Jungblut B, Beis D, Scott IC, D’Amico LA, Ober EA, Verkade H, Field HA, Chi NC, Wehman AM, Baier H, Stainier DYR: A transgene-assisted genetic screen identifies essential regulators of vascular development in vertebrate embryos. Dev Biol 2007;307:29-42.

16 Kleaveland B, Zheng X, Liu JJ, Blum Y, Tung JJ, Zou Z, Chen M, Guo L, Lu MM, Zhou D, Kitajewski J, Affolter M, Ginsberg MH, Kahn ML: Regulation of cardiovascular development and integrity by the heart of glasscerebral cavernous malformation protein pathway. Nat Med 2009;15:169-176.

17 Whitehead KJ, Chan AC, Navankasattusas S, Koh W, London NR, Ling J, Mayo AH, Drakos SG, Marchuk DA, Davis GE, Li DY: The cerebral cavernous malformation signaling pathway promotes vascular integrity via Rho GTPases. Nat Med 2009;15:177-184.
18 Kimmel CB, Ballard WW, Kimmel SR, Ullmann B, Schilling TF: Stages of embryonic development of the zebrafish. Dev Dyn 1995; 203:253-310

19 Liu H, Rigamonti D, Badr A, Zhang J: Ccm1 assures microvascular integrity during angiogenesis. Transl Stroke Res 2010, DOI: 10.1007/s12975-010-0010-Z.

20 Isogai S, Horiguchi M, Weinstein BM: The vascular anatomy of the developing zebrafish: an atlas of embryonic and early larval development. Dev Biol 2001;230:278-301.

21 Isogai S, Lawson ND, Torrealday S, Horiguchi M, Weinstein BM: Angiogenic network formation in the developing vertebrate trunk. Development 2003;130:5281-5290.

22 Davis GE, Black SM, Bayless KJ: Capillary morphogenesis during human endothelial cell invasion of three-dimensional collagen matrices. In Vitro Cell Dev Biol Anim 2000; 36:513-519.

23 Davis GE, Koh W, Stratman AN: Mechanisms controlling human endothelial lumen formation and tube assembly in three-dimensional extracellular matrices. Birth Defects Res C Embryo Today 2007;81:270-285.

24 Kamei M, Saunders WB, Bayless KJ, Dye L, Davis GE, Weinstein BM: Endothelial tubes assemble from intracellular vacuoles in vivo. Nature 2006;442:453-456.

25 Blum Y, Belting HG, Ellertsdottir E, Herwig L, Luders F, Affolter M: Complex cell rearrangements during intersegmental vessel sprouting and vessel fusion in the zebrafish embryo. Dev Biol 2008;316:312-322.

26 Davis GE, Bayless KJ: An integrin and Rho GTPase-dependent pinocytic vacuole mechanism controls capillary lumen formation in collagen and fibrin matrices. Microcirculation 2003;10:27-44.

27 Koh W, Mahan RD, Davis GE: Cdc42- and Racl-mediated endothelial lumen formation requires Pak2, Pak4 and Par3, and PKC-dependent signaling. J Cell Sci 2008;121:9891001.

28 Degani S, Balzac F, Brancaccio M, Guazzone S, Retta SF, Silengo L, Eva A, Tarone G: The integrin cytoplasmic domain-associated protein ICAP-1 binds and regulates Rho family GTPases during cell spreading. J Cell Biol 2002;156:377-388. 